\title{
Excipient selection and aerodynamic characterization of nebulized lipid-based nanoemulsion loaded with docetaxel for lung cancer treatment
}

\begin{abstract}
Docetaxel has demonstrated extraordinary anticancer effects on lung cancer. However, lack of optimal bioavailability due to poor solubility and high toxicity at its therapeutic dose has hampered the clinical use of this anticancer drug. Development of nanoemulsion formulation along with biocompatible excipients aimed for pulmonary delivery is a potential strategy to deliver this poorly aqueous soluble drug with improved bioavailability and biocompatibility. In this work, screening and selection of pharmaceutically acceptable excipients at their minimal optimal concentration have been conducted. The selected nanoemulsion formulations were prepared using high-energy emulsification technique and subjected to physicochemical and aerodynamic characterizations. The formulated nanoemulsion had mean particle size and $\zeta$-potential in the range of 90 to $110 \mathrm{~nm}$ and -30 to $-40 \mathrm{mV}$ respectively, indicating high colloidal stability. The $\mathrm{pH}$, osmolality, and viscosity of the systems met the ideal requirement for pulmonary application. The DNE4 formulation exhibited slow drug release and excellent stability even under the influence of extreme environmental conditions. This was further confirmed by transmission electron microscopy as uniform spherical droplets in nanometer range were observed after storage at $45 \pm 1{ }^{\circ} \mathrm{C}$ for 3 months indicating high thermal stability. The nebulized DNE4 exhibited desirable aerosolization properties for pulmonary delivery application and found to be more selective on human lung carcinoma cell (A549) than normal cell (MRC-5). Hence, these characteristics make the formulation a great candidate for the potential use as a carrier system for docetaxel in targeting lung cancer via pulmonary delivery.
\end{abstract}

Keyword: Docetaxel; Lung cancer; Nanoemulsion; Pulmonary delivery; Sustained release 